\title{
Singular jets during the collapse of drop-impact craters
}

\author{
S. T. Thoroddsen ${ }^{1} \dagger$, K. Takehara ${ }^{2}$, H. D. Nguyen $^{2,3} \&$ T. G. Etoh ${ }^{2}$ \\ ${ }^{1}$ Division of Physical Sciences and Engineering, King Abdullah University of Science and Technology \\ (KAUST), Thuwal, 23955-6900, Saudi Arabia \\ ${ }^{2}$ Department of Civil and Environmental Engineering, Kindei University, Higashi-Osaka 577-8502, Japan \\ ${ }^{3}$ Hanoi University of Science and Technology, No. 1 Dai Co Viet Street, Hanoi, Vietnam
}

(Received xx; revised xx; accepted $\mathrm{xx}$ )

When a drop impacts on a deep pool at moderate velocity it forms a hemispheric crater which subsequently rebounds to the original free-surface level, often forming Worthington jets, which rise vertically out of the crater center. Under certain impact conditions the crater collapse forms a dimple at its bottom, which pinches off a bubble and is also known to be associated with to the formation of a very fast thin jet. Herein we use two ultra-high-speed video cameras to observe simultaneously the dimple collapse and the speed of the resulting jet. The fastest fine jets are observed at speeds of about $50 \mathrm{~m} / \mathrm{s}$ and emerge when the dimple forms a cylinder which retracts without pinching off a bubble. We also identify what appears to be microbubbles at the bottom of this cylinder, which we propose are caused by local cavitation from extensional stress in the flow entering the jet. The radial collapse of the dimple does not follow capillary-inertial power laws nor is its bottom driven by a curvature singularity, as has been proposed in some earlier studies. The fastest jets are produced by pure inertial focusing and emerge at finite dimple size, bypassing the pinch-off singularity. These jets emerge from the liquid contained originally in the drop. Finally, we measure directly the compression of the central bubble following the pinch-off and the subsequent large volume oscillation, which occurs at frequencies slightly above the audible range at about 23 $\mathrm{kHz}$.

\section{Introduction}

\subsection{Crater collapse and singular jets}

Drops impacting on a liquid pool eject a splashing crown, but also form a hemispheric crater. The subsequent rebound of this crater (Fig. 1) tends to focus the energy into a Worthington jet which rises from its center. For low impact velocities the bottom of the crater can form a dimple which pinches off to entrap a small bubble into the pool. This phenomena was studied by Pumphrey \& Elmore (1990) in relation to the underwater sound produced by rain (Prosperetti \& Oguz (1993)). Oguz \& Prosperetti (1990) simulated this phenomenon numerically and suggested bounds in parameter space where this entrapment occurs. The formation of the bottom dimple is connected to capillary waves traveling downwards from the pool surface and focusing at the bottom apex. Similar dynamics occurs for coalescing bubbles (Zhang et al. (2015)). However, the details are strongly dependent on small changes in the impact conditions, with a limited range where very fine high-speed jets are produced. Rein (1996) used a high-speed film camera to study this connection, but only at $500 \mathrm{fps}$. Liow (2001) used $3000 \mathrm{fps}$ to characterize the surface shape for the fastest jets, which he attributed to the retraction of a capillary wave without bubble entrapment. Morton et al. (2000) further characterized the capillary waves and the produced vorticity using numerical simulations. More recently Deng et al. (2007) studied how liquid viscosity and surface tension affect the dimple shapes, showing how the size of the entrapped bubble scales with capillary number. They show that higher viscosity shifts the entrapment regime to larger $W e$. Ray et al. (2015) performed comprehensive simulations of drop-impact crater evolution reproducing many of the observed dimple shapes, while showing jetting speeds less than five times the drop impact velocity. However, their $100 \mu \mathrm{m} /$ grid resolution would not capture the finest jets observed herein.

The most comprehensive experimental study of these jets to date, is that of Michon et al. (2017), 

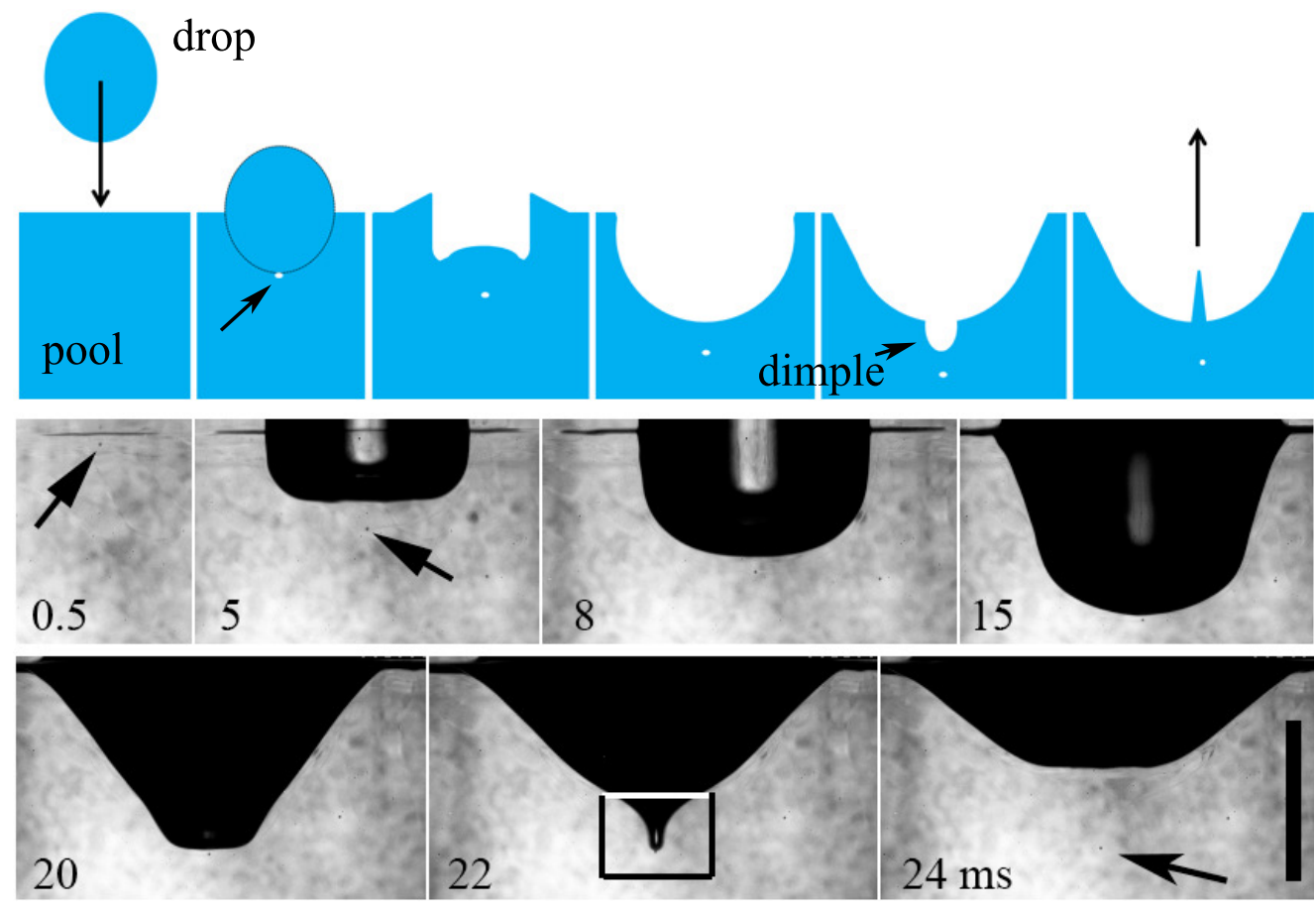

FiguRE 1. Sketch and video frames showing the typical impact crater collapse and jetting. The air crater appears dark under the free surface. Herein we focus the imaging on the dimple, shown inside the rectangle. The arrows point out the tiny bubble entrapped under the drop as it first hits the free surface. The scale bar is $5 \mathrm{~mm}$ long.

who used 8000 fps. They characterize the fast singular jets as "barely reproducible", but observe jet velocities up to 14 times the drop impact velocity.

Zeff et al. (2000) looked at a related problem of crater collapse in a vertically oscillated container, in the context of super-critical Faraday waves. They proposed a self-similar capillary-inertial collapse, where the surface curvature diverges, thereby driving the fast-moving fine jet. On the other hand, they used large-viscosity liquid and their observed jet velocities were larger than the corresponding capillary-viscous velocity $\sigma / \mu_{\ell}$, which does not contain a length scale and should therefore give an upper bound for capillary-driven jets, see Ganan-Calvo (2017). It is therefore unclear what role surface tension and viscosity play, as discussed in Thoroddsen et al. (2007b) who showed the focusing of capillary waves on a free-falling drop can generate a fine jet. Das \& Hopfinger (2008) have investigated the parametrically forced waves in more detail.

\subsection{Jets from bursting bubbles at a pool surface}

Closely related jets are generated by the bursting of bubbles sitting under a free liquid surface. These jets are important for generation of spray above the Ocean surface, which evaporates and turns into aerosols which act as cloud-formation nuclei. Kientzler et al. (1954) performed an early study with a $3000 \mathrm{fps} 16-\mathrm{mm}$ high-speed film camera. MacIntire (1972), Boulton-Stone \& Blake (1993) and Duchemin et al. (2002) performed numerical simulations of this jetting process.

Renewed interest is this phenomenon has been driven by commercial beverage applications. The crater jets are clearly visible rising from the surface of soft drinks or champagne, producing the familiar fizzy sound of freshly poured beverages. The details are this effervescence are of great importance to the sparkling wine industry Séon \& Liger-Belair (2017). However, for the popping bubbles the crater size is limited to the capillary length (Ghabache et al. (2014); Walls et al. (2015)) which is significantly smaller than the impact craters studied herein. The scaling of the jetting is therefore dependent on the Bond number, as recently discussed by Krishnan et al. (2017). On the other hand Ganan-Calvo (2017) has scaled the size of the principal drop pinched from the jet tip, in a universal manner, using a capillary-inertial scaling in terms of an Ohnesorge number, without contribution from gravity.

Deike et al. (2018) have performed the most detailed simulation of these bubble-bursting jets to 
(a)

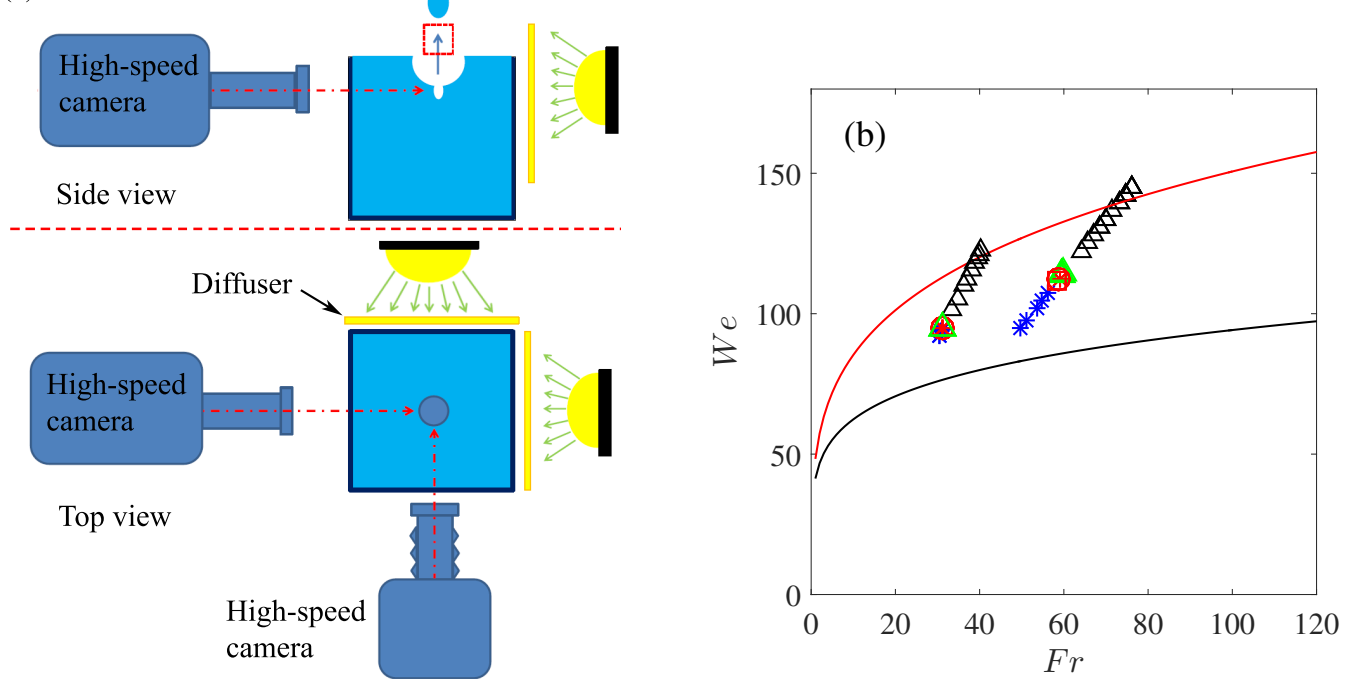

FIGURE 2. (a) Sketch of the experimental setup, with two high-speed video cameras viewing the dynamics from perpendicular directions. One camera captures the dimple dynamics inside the pool, while the other views the jet droplets as they emerge out of the crater (within red square). (b) Our impact conditions in $\mathrm{Fr}-W e$-space compared to the bounds of the range of the regular bubble entrapment regime measured by Pumphrey \& Elmore (1990), using the best fit curves from Oguz \& Prosperetti (1990). The two linear clusters of points correspond to different drop diameters of $D=3.3 \mathrm{~mm}$ and $4.2 \mathrm{~mm}$, with the larger drop at lower Fr. The meaning of the symbols are given graphically in Fig. 3.

date. They include both viscosity and gravity and present the results with the Bond and Laplace numbers. They find a narrow range of parameters where viscosity cuts off the self-similar collapse of the capillary wave (Duchemin et al. (2002)) and the dynamics develop a finite-time singularity, where they suggest surface curvature and pressure diverge.

The phenomenon of hand-held fireworks has been shown to be related, as jetting develops from gas bubble nucleation and bursting within the melt (Inoue et al. (2013)). This can lead to a cascade of incandescent jets from the subsequent satellite droplets (Inoue et al. (2017)).

\subsection{Open questions}

Despite decades of study, key open questions remain on the underlying dynamics of the fine singular crater jets. Rein (1996) as well as Michon et al. (2017) note that fast jets can occur with or without the dimple pinching off a central bubble. Liow (2001) and Zeff et al. (2000) propose the fastest jets are formed by capillary retraction of the dimple without pinch-off. None of these studies had imaging with sufficient time-resolution to resolve this issue. Herein we use unprecedented time and space resolution to pinpoint new aspects of these phenomena. Using $2 \mu \mathrm{s}$ time resolution, not available to earlier studies, we show that while fast jets are generated by dimple pinch-off, the very fastest jets occur by pure inertial focusing when the dimple does not pinch off, but its bottom rises vertically at high speed. No curvature singularity develops and the jet passes through the dimple. The dynamics thereby bypass the pinch-off singularity.

\section{Experimental Setup}

\subsection{Imaging setup}

The experimental configuration is sketched in Figure 2(a). The drop is released from a millimetric flat steel nozzle and subsequently free-falls onto a pool surface, contained in a square plexiglass tank. The liquid is $\sim 6.5 \mathrm{~cm}$ deep inside of $9.4 \times 9.4 \mathrm{~cm}$ inner cross-section. The drop diameters are less than $4.2 \mathrm{~mm}$, so we do not expect capillary waves reflected from the walls to influence the crater collapse.

We use two synchronized ultra-high-speed video cameras (Etoh et al. (2003)), made by Shimadzu Corp., one was a prototype and the other a production model. One camera is used to observe the crater evolution below the free surface and the other to catch the fine jetting as it emerges from 
the crater and rises above the free surface. The two cameras are arranged perpendicularly to each other, with each having a separate backlight diffuser. These cameras can take 102 frames at frame rates up to 1 million fps, each with $260 \times 312 \mathrm{px}$, irrespective of the frame-rate. The imaging was triggered by the falling drop cutting the beam from a laser-pointer, which shone on a photodiode. The two camera time-delays were adjusted independently, to catch the relevant feature on the two video clips.

We use a long-distance microscope (Leica Z16 APO) for imaging the crater evolution. It has adjustable magnification and aperture. Magnifications as large as 14.6 were used herein, giving a corresponding pixel resolution of up to $2.98 \mu \mathrm{m} / \mathrm{px}$. This required intense back-lighting with a Sumita metal-halide lamp, shone on a diffuser. This camera used frame-rates as high as $500 \mathrm{kfps}$, which is in practise limited by the jitter in the trigger timing.

The other camera, used to observe the jetted fine droplets, had a $200 \mathrm{~mm}$ Nikon lens with bellows to provide added magnification, for resolution of $20.3 \mu \mathrm{m} / \mathrm{px}$. To capture the fastest jetting we use frame-rates as high as $200 \mathrm{kfps}$.

\subsection{Liquids and drop shapes}

We use the same $50 \%$ by-volume water/glycerin mixture for the drop and pool liquids. The pool and air temperatures were measured continuously with imbedded thermometers and were in the range 22 to $26^{\circ} \mathrm{C}$, which was taken into account when calculating liquid properties. For typical temperatures $T \simeq 24^{\circ} \mathrm{C}$, this gives liquid density of $\rho=1140 \mathrm{~kg} \cdot \mathrm{m}^{-3}$, dynamic viscosity $\mu=7.13 \times 10^{-3}$ Pa.s and the corresponding kinematic vicosity $\nu=6.25 \times 10^{-6} \mathrm{~m}^{2} \mathrm{~s}^{-1}$. The surface tension is $\sigma=0.065 \mathrm{~N} . \mathrm{m}^{-1}$. The illumination is blocked between impacts, but minor temperature difference can arise from the intense lighting and cause slight difference in the index of refraction between drop and pool, when viewing tangent along their interface. This becomes visible as a faint line separating the two liquids in the video images.

Some initial experiments were performed with deionized water, but the craters were too variable to allow for repeatable trigger timing, which is needed for the short-duration video clips of our cameras.

The drop is pinched off from one of two tapered stainless steel nozzles with a sharp inner edge of $\sim$ 1.2 or $1.8 \mathrm{~mm}$ in diameter, producing droplets with diameters of 3.33 and $4.20 \mathrm{~mm}$ respectively. The impact velocities and droplet shapes were measured from the top camera. The elevated viscosity from that of water, quickly reduces oscillations from the pinch-off during the free-fall, with the drop being axisymmetric. However, some shape-oscillations remain which we characterize by giving the aspect ratio of $\alpha=D_{V} / D_{H}$, where $D_{H}$ is the horizontal and $D_{V}$ the vertical diameters at impact. The equivalent spherical drop diameter is estimated as $D=\left(D_{V} D_{H}^{2}\right)^{1 / 3}$. The aspect ratio varies between 0.95 to 1.05 . The release height $H$, measured from the nozzle to the pool surface, was varied from $H \simeq 6-12 \mathrm{~cm}$, giving impact velocities of between $U \simeq 1.1-1.6 \mathrm{~m} \mathrm{~s}^{-1}$.

\subsection{Parameter Space}

We characterize the impact conditions by the Reynolds, Weber and Froude numbers,

$$
R e=\frac{\rho D U}{\mu}, \quad W e=\frac{\rho D U^{2}}{\sigma}, \quad F r=\frac{U^{2}}{g D},
$$

where $g$ is the acceleration of gravity. Note that this definition of $F r$, without the square-root, is used in the relevant earlier studies on this topic. Figure 2(b) shows where our impact conditions fall in the classical regime constructed by Pumphrey \& Elmore (1990) and fitted by Oguz \& Prosperetti (1990), which shows where the regular entrapment of a bubble at the crater center occurs. Our data overlap, on the upper $W e$ range, but no bubble entrapment occurs for our blue stars. This discrepancy is not surprising, as our liquid is more viscous than in those studies. This is consistent with the trends observed by Deng et al. (2007). Our Re are always in the range from 700 to 900 . 
(a)
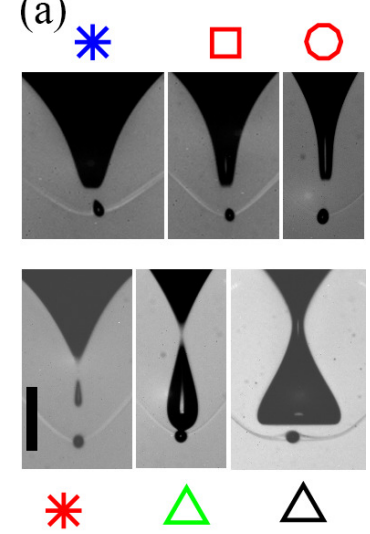
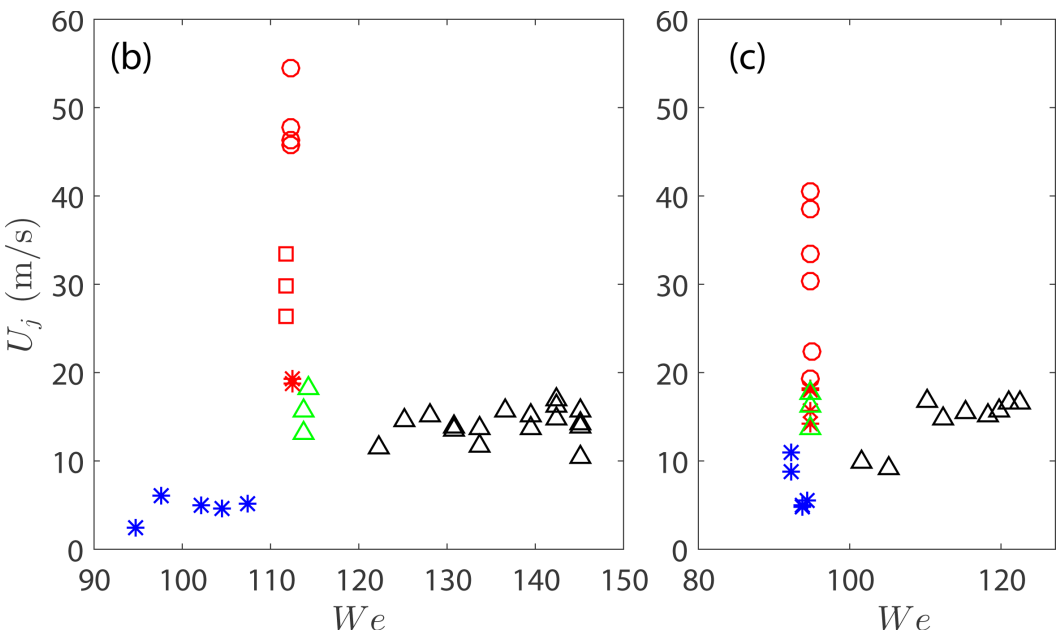

Figure 3. Jetting velocity $U_{j}$ vs impact $W e$ spanning the range of dimple collapse. (a) The symbols corresponding to different dimple shapes. Scale bar is $500 \mu \mathrm{m}$. No pinch-off shallow angled dimple $\left({ }^{*}\right)$. Weakly singular jet $(\square)$. Singular jet $(\circ)$. Small bubble pinched off $(*)$. Teardrop pinch-off $(\triangle)$. Conical dimple pinch-off $(\triangle)$. (b,c) Jet velocities vs impact $W e$, for two different drop sizes of $D=3.33 \mathrm{~mm}$ in (b) and for $D=4.20 \mathrm{~mm}$ in (c).

\section{Results}

\subsection{Dimple shapes and jetting speeds}

We focus on the crater collapse when a dimple forms at its bottom and fine jets are produced. Figure 3(a) shows how we categorize the dimple shapes which appear. During the crater collapse capillary waves travel from the rim down towards the bottom center. They can form conical shapes without pinching off a bubble $(*)$. As we increase the impact velocity these cones become sharper and eventually form a narrow vertical cylinder, which produces what we term singular jetting (o). This occurs in a narrow range of $U$ or $W e$, shown in Fig. 3. Past this regime we observe regular pinch-off of large bubbles with $D_{b} \sim 300-500 \mu \mathrm{m}$. We split this into teardrop $(\triangle)$ and triangular pinch-off $(\triangle)$. While the singular jets occur at almost the same $W e$ as the small-bubble pinch-off (*) there is no ambiguity regarding the jetting speed for each case, as we have simultaneous viewing of the jet and the dimple.

Note that the small bubble of $\sim 80 \mu \mathrm{m}$ in diameter, sitting under the dimple in Figs. $3 \& 4$, is due to the air-disc entrapped under the center of the drop, when it first contacts the pool, as was studied by Thoroddsen et al. (2003). This bubble sits along the faint line separating the drop and pool liquids (see section 2.2). It is telling about the image resolution of earlier studies, that this unavoidable central entrapped bubble is not visible in most of them.

Panels (b) \& (c) in Fig. 3 show how the jetting velocity $U_{j}$ changes for the different dimple pinch-off shapes, as we increase $U$ and thereby $W e$. Both drop diameters show the same trend. These velocities are measured from the first droplets which emerge out of the crater, which we presume originate from the tip of the narrow jet, as it breaks up by Rayleigh instability. For the fast jets we typically see 20 micro-droplets. $U_{j}$ spikes at a narrow range of $W e$, where it reaches four-fold velocity compared to the surrounding conditions. This higher velocity coincides with the singular cylinder, where no bubble is pinched off. For the smaller drop this occurs at $W e=112$ and at $W e=95$ for the larger drop. The corresponding values of $F r \simeq 60$ and 30 is much smaller than other studies, see Michon et al. (2017).

Figure 4 shows the details of the dimple collapse and pinch-off. The sequences of frames have time-resolution of 2 or $4 \mu \mathrm{s}$. The necking collapse in Fig. 4(a) produces two fine Worthington jets, which travel up and down from the neck. The downwards traveling jet penetrates the bottom of the bubble, in the 7th image, as is marked by the arrow. We can estimate the speed of this downwards jet, by measuring the distance from the neck to the bottom of the bubble and dividing by the $25 \mu \mathrm{s}$ duration of this travel. This gives a repeatable velocity over a few realizations, of $u_{j}=36.2 \pm 4.2$ $\mathrm{m} / \mathrm{s}$. We estimate the width of this jet to be roughly $\sim 15 \mu \mathrm{m}$. Keep in mind that the flat bottom of the bubble shadow, does not mean the bubble is flat and indeed the bottom appears to be bent 
6

Thoroddsen, Takehara, Nguyen E Etoh
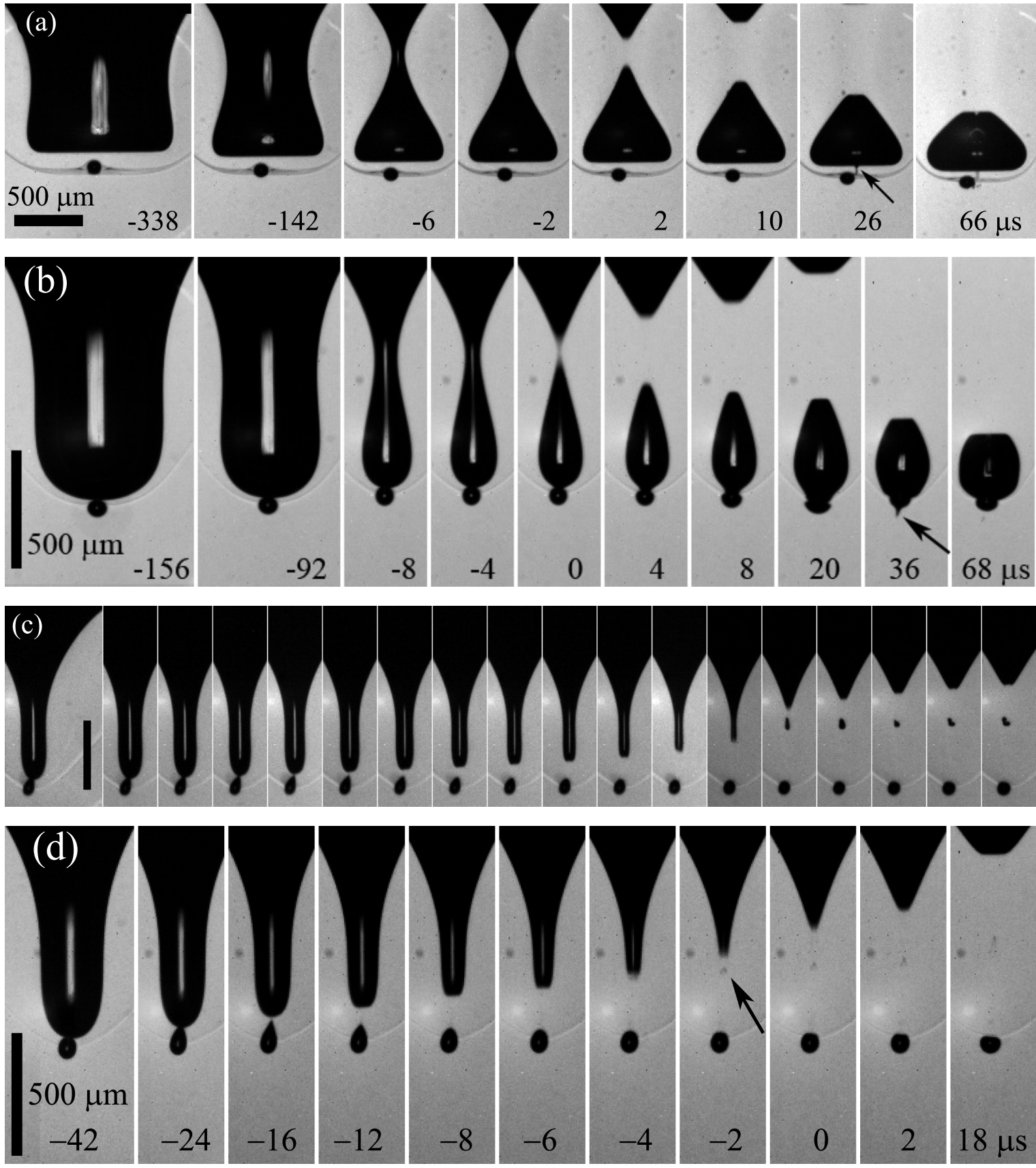

Figure 4. Typical dimple collapse for different impact conditions. The small bubble sitting below the dimple at the start of each sequence, is the central bubble entrapped under the drop as it first impacts the pool, see Thoroddsen et al. (2003). (a) Triangular necking. The arrow points out a fine jet, produced by the pinch-off, which penetrates through the bottom of the bubble. $U=1.27 \mathrm{~m} / \mathrm{s}, D=4.20 \mathrm{~mm}, W e=118$, $F r=39 \& R e=895$. (b) Teardrop necking. The arrow points at the penetrating downwards jet. $U=1.40$ $\mathrm{m} / \mathrm{s}, D=3.33 \mathrm{~mm}, W e=114, F r=60 \& R e=783$. (c) Cylindrical dimple collapse, with a small bubble pinched off. The pinched-off bubble splits into two small bubbles. The time between frames is $2 \mu \mathrm{s}$ and scale bar is $500 \mu \mathrm{m} . U=1.13 \mathrm{~m} / \mathrm{s}, D=4.20 \mathrm{~mm}, W e=95, F r=31 \& R e=801$. (d) Cylindrical dimple singular collapse. The arrow points out microbubbles formed at the end of the rapidly moving bottom. $U=1.39 \mathrm{~m} / \mathrm{s}, D=3.33 \mathrm{~mm}, W e=112, F r=59 \& R e=776$. See also supplementary Videos $1-4$.

upwards at the centerline. Similar jet speeds are seen for the teardrop pinch-off in panel (b). Panel (c) shows a near-singular pinch-off where only a small bubble is pinched off. Figure 3 showed that any pinch-off reduces greatly the jetting velocity, even the tiny bubble in Fig. 4(c). Finally, panel (d) shows singular retraction of the tip, which corresponds to the largest jetting velocities. 

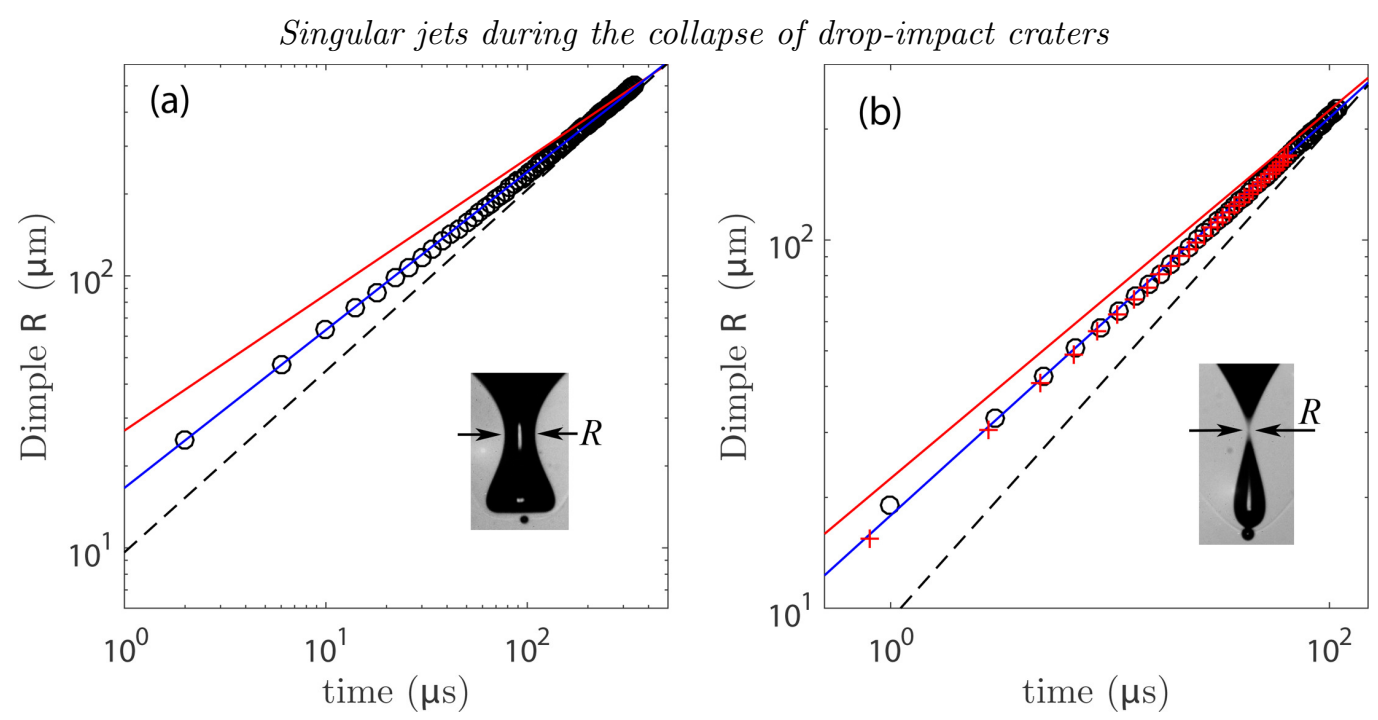

Figure 5. Radial collapse of the dimple pinch-off. The dashed lines correspond to capillary-inertial dynamics with a slope of $2 / 3$, while the solid red lines correspond to inertial focusing at slope of $1 / 2$. (a) The pinch-off of a conical dimple, with an exponent of $\beta=0.58 \pm 0.02$. (b) Two realizations for the radius of teardrop pinch-off from $500 \mathrm{kfps}$, giving power-law exponent near pinch-off of $\beta=0.54 \pm 0.02$ (blue line).

The faint line visible in the images separates the drop and pool liquids and arises most likely from slight differences in temperature between the two, as explained in sec. 2.2. This shows that the fast jets contain only drop liquid, see Gekle \& Gordillo (2010).

\subsection{Power-laws for collapse of dimple radius}

The final pinch-off of the dimple was recorded at up to $500 \mathrm{kfps}$, which allows us to track the dimple radii vs time to reveal the power-law behaviour near the final pinch-off,

$$
R(t)=C\left(t_{o}-t\right)^{\beta}
$$

where $t_{o}$ is our estimate of the pinch-off time. $C$ is a fitting parameter, which becomes independent of $\sigma$ and gravity at the smallest scales. For the conical dimple we follow the minimum radius, whereas for the teardrop pinch-off we follow the radius at the depth of the final pinch-off. Figure 5 shows that for both cases the exponents are closer to $1 / 2$ for inertial collapse, than to the capillaryinertial dynamics, which should follow a slope of $2 / 3$. The values of the exponents are close to those observed for bubble pinch-off from rest in Thoroddsen et al. (2007a).

Figure 6(a) traces the radial collapse and vertical retraction of the singular cylindrical dimple, like the one shown in Fig. 4(d). Here the dimple has an almost vertical wall, so its radius is well-defined. In other cases the wall is more cone-shaped and we measure the radius at a fixed $z$-location, where the maximum vertical retraction speed $d z / d t$ occurs, which we also select as time $t_{o}$. When the cylinder becomes narrowest its bottom moves up most rapidly, but its width does not go through a singularity, rather reaching here a minimum radius of $R \simeq 11 \mu \mathrm{m}$, which we propose coincides with the formation of the vertical fine jet. Following this the edge of the cylinder widens again at a slow rate. Figure $6(\mathrm{~b})$ shows that the radius again follows an inertial power-law with exponent $\beta=0.53 \pm 0.02$, with the caveat that selecting $t_{o}$ is somewhat arbitrary in this case.

The arrow in Fig. 4(d) points at a faint group of micro-bubbles separating from the tip of the air-cylinder. Figure 7 shows close-up images of this phenomenon, where the rapidly pulled up air cylinder leaves behind a small conical region of micro-bubbles, pointed out with the arrows. This dark feature has characteristic conical shape with its apex reaching into the jet base, pointing its direction. We suggest this structure forms slightly behind the jet which itself is driven by the high pressure from the inertial focusing. Immediately following this ejection the flow which turns sharply around the jet base, as is sketched in panel (c), could separate generating low pressure and cavitate the liquid. This micro-bubble signature is faint, but it occurs in a repeatable fashion. 

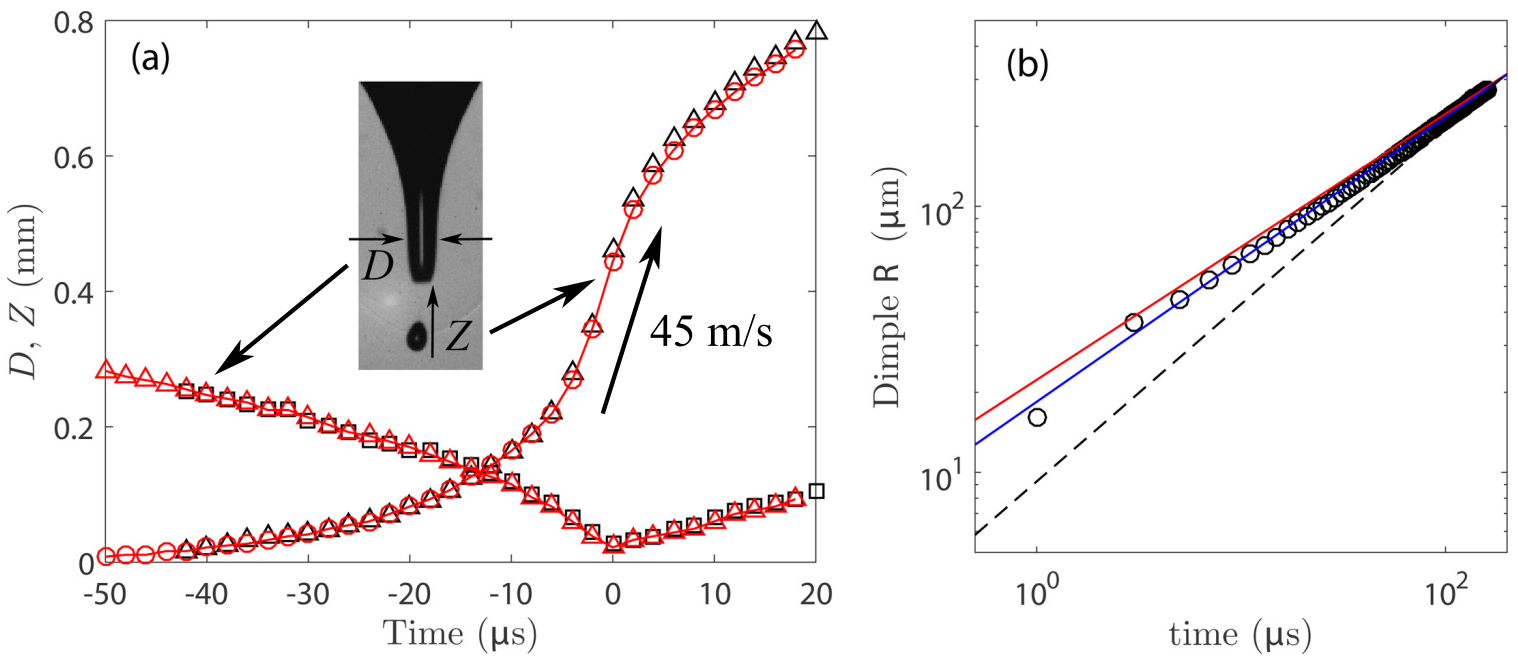

Figure 6. Radial collapse of the singular dimple cylinder. (a) The diameter of the tip and the vertical motion of the bottom $Z$ of the air cylinder. (b) The radius of bottom cylinder taken from from $500 \mathrm{kfps}$ video. The best fit line gives a power-law exponent near pinch-off of $\beta=0.535 \pm 0.02$ (blue line). The dashed line corresponds to capillary-inertial dynamics with a slope of $2 / 3$, while the solid red line corresponds to inertial focusing of slope $1 / 2$.

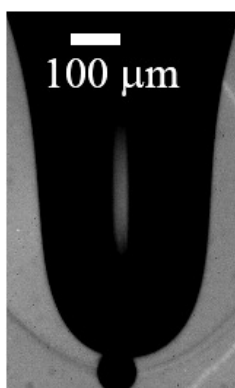

0

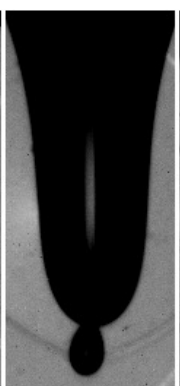

40

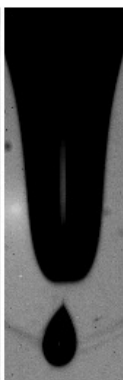

56

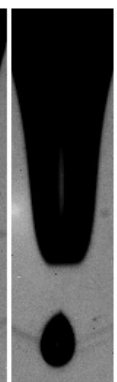

60

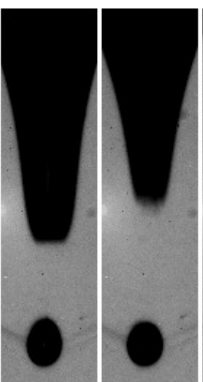

$64 \quad 68$

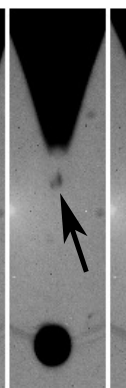

72

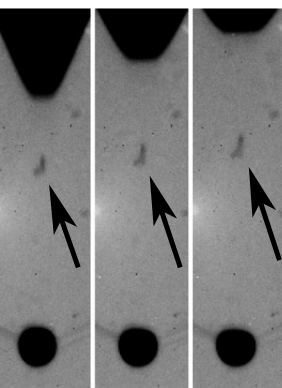

$76 \quad 80 \quad 84 \mu \mathrm{s}$

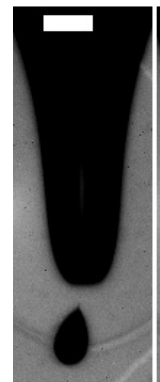

0

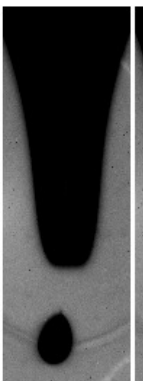

4

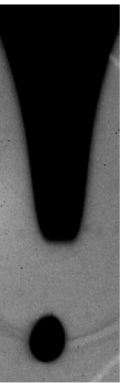

8

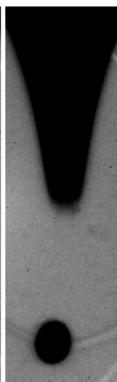

12

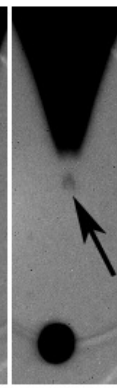

16

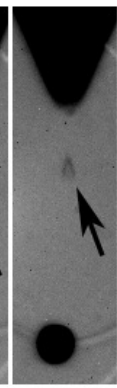

$20 \quad 32 \mu \mathrm{s}$

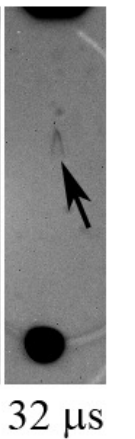

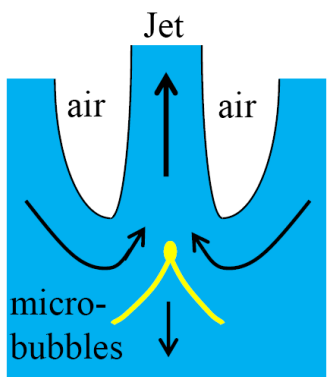

Figure 7. Two realizations showing rapidly retracting cylindrical bottom dimple, shedding micro-bubbles, as pointed out by the arrows. The scale bars are both $100 \mu \mathrm{m}$ long. See also supplementary Videos $5 \& 6$.

\subsection{Bubble compression and sound generation}

Note the sudden apparent volume expansion of the bubble, in Fig. 4(b), between times 4 and $20 \mu$ s after the pinch-off. The supplemental videos $2 \& 7$ show clearly the subsequent cycles of compression and expansion of the bubble. If we assume axisymmetry of the bubble and ignore the volume of the fine jet we can obtain a rough estimate of the volume compression. This ratio varies widely, but in some cases it exceeds a factor of 2.5, as in the case shown in Fig. 8 when comparing frames 5 and 7 . The amplitude in subsequent oscillations will rapidly reduce by viscous dissipation. This suggests excess pressure above atmospheric. The oscillations of this bubble has indeed been shown to be the source of the underwater sound of rain on the Ocean (Pumphrey \& Elmore 


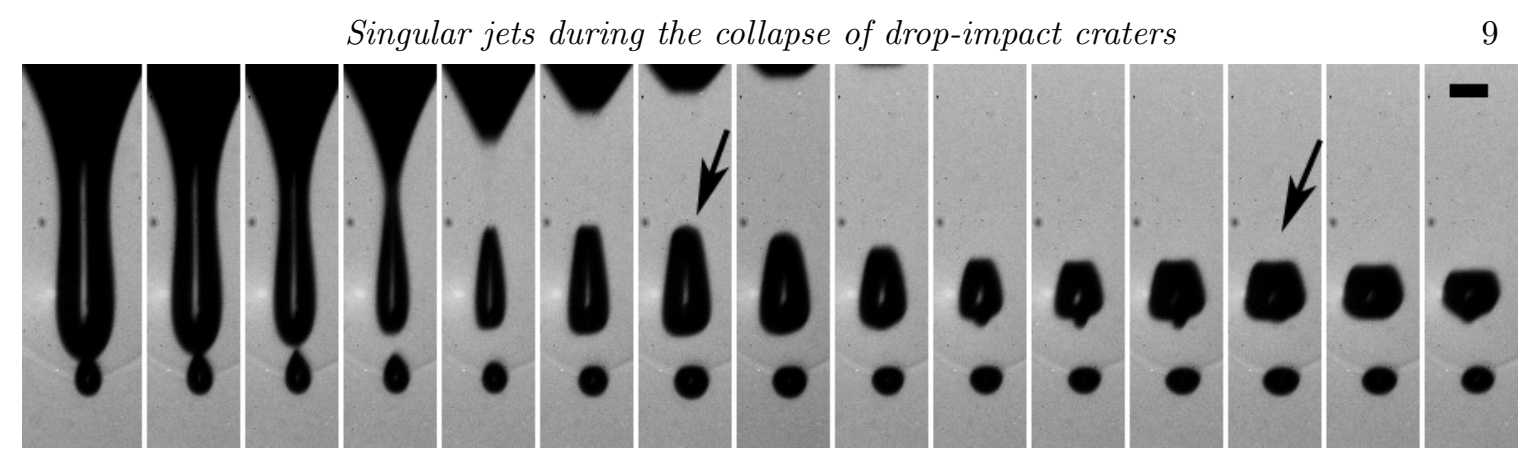

Figure 8. Compression of the pinched-off bubble. Time between frames is $4 \mu$ s and the scale bar is $100 \mu \mathrm{m}$ long. The arrows point out the maximum bubble volume during the expansion phase. See also supplementary Video 7.

(1990); Oguz \& Prosperetti (1990, 1991); Prosperetti \& Oguz (1993)), using hydrophones. Here we can measure the oscillation frequency directly from the video. From numerous such identical measurements we obtain $23 \pm 2 \mathrm{kHz}$, which is slightly above the audible range which reaches 20 $\mathrm{kHz}$. The rain-produced sound energy in the ocean has a peak at $14 \mathrm{kHz}$, see Prosperetti \& Oguz (1993). Keep in mind that the bubble diameter in Fig. 8 is only about $170 \mu \mathrm{m}$ and frequency will be inversely proportional to size. However, this points out the potential power of this direct imaging, which could be acquired along-side hydrophone records.

\section{Discussion and Conclusions}

We conclude that the most singular jets arise from inertia focusing, without bubble pinch-off. This is consistent with the seminal results of Zeff et al. (2000), who observed their fastest jets without pinch-off. However, the free-surface dynamics are not self-similar and divergent surface tension is not the driving mechanism, as they suggested and was explained by Brenner (2000). See also the follow-up study by Das \& Hopfinger (2008) who suggest inertial focusing, but did not have time-resolved imaging to capture the fastest jets. We further verify this by tracking the collapse of the final dimple radius, in Fig. 6 , which reveals power-law $\simeq 0.55$ with close to the pure inertial exponent of $1 / 2$ as is observed for bubble pinch-offs (Burton et al. (2005); Keim et al. (2006); Thoroddsen et al. (2007a)). Indeed measuring values slightly larger than 0.5 is expected, as the transition in the value of the exponent from capillary-driven to inertial-driven dynamics, in bubble pinch-off, is known to be logarithmically slow (Gordillo et al. (2005); Bergmann et al. (2006); Eggers et al. (2007)).

The finest jets are much thinner and faster than would have been possible to observe in most of the previous studies. Here we observe the fastest droplets jetting at velocities $\sim 50 \mathrm{~m} / \mathrm{s}$ when they emerge out of the crater, while Deng et al. (2007) saw a maximum of $15 \mathrm{~m} / \mathrm{s}$, Ghabache et al. (2014) see $14 \mathrm{~m} / \mathrm{s}$ and Liow (2001) measured $10.5 \mathrm{~m} / \mathrm{s}$. These are certainly all underestimates, as the very small tip droplets are greatly decelerated by air drag before they emerge out of the crater and the initial speed of the jets will therefore be even larger. Typical deceleration is shown for a representative droplet in the Supplemental materials. This explains why the early downwards jets for the teardrop pinch-off, in Fig. 4(b), are much faster $(35 \mathrm{~m} / \mathrm{s})$ than those emerging out of the crater at $19 \mathrm{~m} / \mathrm{s}$. Extrapolating this, the singular jets may be generated at closer to $100 \mathrm{~m} / \mathrm{s}$. Observations by X-rays, of the jet inside the dimple, would be very useful but are currently not available at such high frame-rates (Zhang et al. (2012)). Pinning down the diameter and speed of the very finest droplets will require even finer time and space resolution.

Intuitively, one might expect the radial collision of the dimple walls to produce the fastest jets, in a similar way as for the fine Worthington jets produced by the collapse of sphere-impact cavities (Worthington \& Cole (1897); Gekle et al. (2009); Gekle \& Gordillo (2010)). But this is not the case in our configuration. Figure 9 graphically summarizes the jetting speed results. The fastest jets occur at the lower bound of the bubble pinch-off regime. At $W e$ below this boundary we find broader conical dimples with much slower jetting. Closer to the first pinch-off the dimple cylinder becomes steeper forming faster and faster jets. Once pinch-off occurs the jetting speed drops greatly. In essence the flow bypasses a singularity, but tries to get as close as possible. This leaves open the question, whether there exists an even finer tuning which will produce still faster 


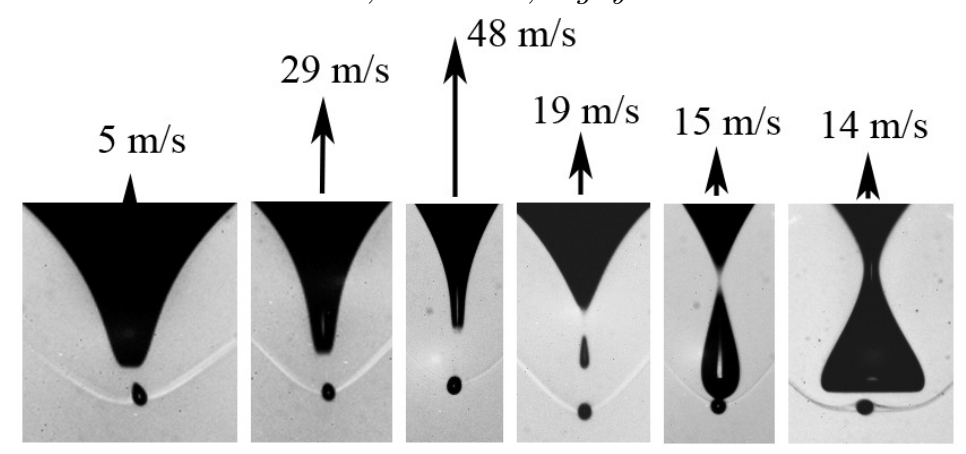

FiguRE 9. Schematic of jetting speeds vs dimple shapes, corresponding to Fig. 3. The arrow lengths indicate the jet velocities. The Weber number grows from left to right.

jets, or will this be mitigated by cavitation? The sketch in Fig. 7 indicates the overall flow field at the base of the jet, which contains a stagnation point with high pressure required to drive the jet through the bottom of the dimple, see also sketch in Ganan-Calvo (2017). Figure 8 also underscores the large induced pressure by the very strong compression of the gas, observed when the bottom dimple is pinched off. While the inertial focusing will produce high pressure at the base of the dimple to drive the fine jet, Fig. 7 shows intriguing clues for extreme suction pressure in the region near the base of the jet, by the formation of micro-bubbles. These bubbles could be pulled from the free surface, but we speculate cavitation could be at play. The flow is extremely transient and the rapid motions of a curved free surface are known to generate vorticity which can play a role here, as shown by Thoraval et al. (2012). Indeed, the very strong extensional forces, suggested by the formation of these micro-bubbles in Fig. 7, may be important for damaging cells in bioreactors when bubbles pop at free surfaces, as has been suggested by Tran et al. (2016) and Walls et al. (2017).

There is clearly need for further study of the details of the flow-fields during the dimple collapse with fully-resolved simulations and theory, in the vein of Gekle \& Gordillo (2010); Morton et al. (2000); Ray et al. (2015); Walls et al. (2015); Deike et al. (2018).

In summary, herein we have shown that the fastest thin jets bypass the dimple pinch-off singularity and are driven purely by inertial focusing.

The work reported herein was partly funded by King Abdullah University of Science and Technology (KAUST).

\section{REFERENCES}

Bergmann, R., van der Meer, D., Stijnman, M., Sandtke, M., Prosperetti, A. \& Lohse, D., 2006 Giant bubble pinch-off, Phys. Rev. Lett. 96, 154505.

Boulton-Stone, J. M. \& Blake, J. R. 1993 Gas bubbles bursting at a free surface. J. Fluid Mech. 254, $437-466$

Brenner, M. P. 2000 Jets from a singular surface, Nature, 403, 377.

Burton, J. C., Waldrep, R. \& Taborek, P., 2005 Scaling and instabilities in bubble pinch-off, Phys. Rev. Lett. 94, 184502.

DAs, S. P. \& Hopfinger, E. J. 2008 Parametrically forced gravity waves in a circular cylinder and finite-time singularity. J. Fluid Mech. 599, 205-228.

Deike, L., Ghabache, E., Liger-Belair, G., Das, A. K., Zaleski, S., Popinet S. \& Séon, T. 2018 Dynamics of jets produced by bursting bubbles, Phys. Rev. Fluids, 3, 013603.

Deng, Q., Anilkumar, V. \& Wang, T. G. 2007 The role of viscosity and surface tension in bubble entrapment during drop impact onto a deep liquid pool. J. Fluid Mech. 578, 119-138.

Duchemin, L., Popinet, S., Josserand, C. \& Zaleski, S. 2002 Jet formation in bubbles bursting at a free surface, Phys. Fluids, 14, 3000.

Eggers, J., Fontelos, M. A., Leppinen, D. \& Snoeijer, J. 2007 Theory of the collapsing axisymmetric cavity, Phys. Rev. Lett., 98, 094502.

Етон, T. G. et al. 2003, An image sensor which captures 100 consecutive frames at 1000000 frames/s, IEEE Trans. Electron Devices 50, 144-151.

Ganan-Calvo, A. M. 2017 Revision of bubble bursting: Universal scaling laws of top jet drop size and speed, Phys. Rev. Lett., 119, 204502. 
Gekle, S., Gordillo, J. M., van Der Meer, D. \& Lohse, D. 2009 High-speed jet formation after solid object impact, Phys. Rev. Lett., 102, 034502.

Gekle, S. \& Gordillo, J. M. 2010 Generation and breakup of Worthington jets after cavity collapse. Part 1. Jet formation, J. Fluid Mech., 663, 293-330.

Ghabache, E., Antkowiak, A., Josserand, C. \& SÉon, T. 2014 On the physics of fizziness: How bubbles bursting controls droplets ejection, Phys. Fluids, 26, 121701.

Gordillo, J. M., Sevilla, A., Rodríguez-Rodríguez, J. \& C. Martínez-BazÁn, 2005 Axisymmetric bubble pinch-off at high Reynolds numbers, Phys. Rev. Lett., 95, 194501.

Inoue, C., Koshi, M., Terashima, H., Himeno, T. \& Watanabe, T. 2013 Origin of droplets in sparkling fireworks, Sci. Technol. Energetic Mater. 74, 106-111.

Inoue, C., Izato, Y., Miyake, A. \& Villermaux, E. 2017 Direct self-sustained fragmentation cascade of reactive droplets. Phys. Rev. Lett., 118, 074502.

Keim, N. C., Moller, P., Zhang, W. W. \& Nagel, S. N. 2006 Breakup of Air Bubbles inWater: Memory and Breakdown of Cylindrical Symmetry. Phys. Rev. Lett., 97, 144503.

Kientzler, C. F., Arons, A. B., Blanchard, D. C. \& Woodcock, A. H. 1954 Photographic investigation of the projection of droplets by bubbles bursting at a water surface. Tellus, 6, 1-7.

Krishnan, S., Hopfinger, E. J. \& Puthenveettil, B. A. 2017 On the scaling of jetting from bubble collapse at a liquid surface, J. Fluid Mech., 822, 791-812.

Liow, L. J. 2001 Splash formation by spherical drops, J. Fluid Mech., 427, 73.

MacIntiRe, F. 1972 J. Geophys Res. 77, 5211.

Michon, G.-J., Josserand C. \& SÉon, T. 2017 Jet dynamics post drop impact on a deep pool, Phys. Rev. Fluids, 2, 023601.

Morton, D., Rudman, M. \& Liow, J.-L. 2000 An investigation of the flow regimes resulting from splashing drops, Phys. Fluids 12, 747-763.

Oguz, H. N. \& Prosperetti, A., 1990 Bubble entrainment by the impact of drops on liquid surfaces, J. Fluid Mech. 219, 143-179.

Oguz, H. N. \& Prosperetti, A., 1991 Numerical calculation of the underwater noise of rain , J. Fluid Mech. 228, 417-442.

Prosperetti, A. \& Oguz, H. N., 1993 The impact of drops on liquid surfaces and the underwater noise of rain, Annu. Rev. Fluid Mech. 25, 577-602.

Pumphrey, H. C. \& Elmore, P. A. 1990 The entrainment of bubbles by drop impacts, J. Fluid Mech., $\mathbf{2 2 0}, 539$.

Ray, B., Biswas, G. \& Sharma, A. 2015 Regimes during drop impact on a liquid pool, J. Fluid Mech., 768, 492-523.

ReIN, M. 1996 The transition regime between coalescing and splashing drops, J. Fluid Mech., 306, 145.

SÉon, T. \& Liger-Belair, G. 2017 Effervescence in champagne and spartling wines: From bubble bursting to droplet evaporation, Eur. Phys. J. Special Topics, 226, 117-156.

Thoraval, M.-J., Takehara, K., Etoh, T. G., Popinet, S., Ray, P. Josserand, C., Zaleski, S. \& Thoroddsen, S. T. 2012 Von Kármán vortex street within an impacting drop, Phys. Rev. Lett., 108, 264506.

Thoroddsen, S. T., Takehara, K. \& Eтон, T. G. 2003 Air entrapment under an impacting drop, J. Fluid Mech. 478, 125-134.

Thoroddsen, S. T., Eтон, T. G. \& Takehara, K. 2007a, Experiments on bubble pinch-off. Phys. Fluids, 19, 042101.

Thoroddsen, S. T., Етон, T. G. \& Takehara, K. 2007b, Micro-jetting from wave-focusing on oscillating drops. Phys. Fluids, 19, 052101.

Tran, T. T., Lee, E. G., Lee, I. S., Woo, N. S., Han, S. M., Kim, Y. J. \& Hwang, W. R. 2016 Hydrodynamic extensional stress during the bubble bursting process for bioreactor system design, Korea-Aust. Rheol. J., 28, 315-326.

Walls, P. L. L., Henaux, L. \& Bird, J. C. 2015 Jet drops from bursting bubbles: How gravity and viscosity couple to inhibit droplet production, Phys. Rev. E, 92, 021002(R).

Walls, P. L. L., Mcrae, O., Natarajan, V., Johnson, C. Antoniou, C. \& Bird, J. C. 2017 Quantifying the potential for bursting bubbles to damage suspended cells Sci. Reports, 7, 15102.

Worthington, A. M. \& Cole, R. S. 1897 Impact with a Liquid Surface, Studied by the Aid of Instantaneous Photography. Phil. Trans. R. Soc. A. 189, 137-148.

Zeff, B. W., Kleber, B., Fineberg, J. \& Lathrop D. P. 2000 Singularity dynamics in curvature collapse and jet eruption on a fluid surface, Nature (London) 403, 401.

Zhang, L. V., Toole, J., FezzaA, K. \& Deegan, R. D. 2012 Evolution of the ejecta sheet from the impact of a drop with a deep pool. J. Fluid Mech. 690, 5-15.

Zhang, F., Thoraval, M.-J., Thoroddsen, S. T. \& Taborek, P. 2015 Partial coalescence from bubbles to drops, J. Fluid Mech., 782, 209-239. 